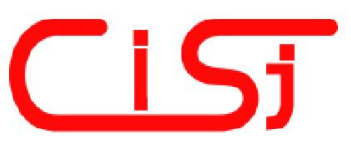

\title{
PROGRAMMABLE PARALLEL FBD SIGMA DELTA ADC RECONSTRUCTION STAGE DESIGN FOR SOFTWARE DEFINED RADIO RECEIVER
}

\author{
Rihab Lahouli ${ }^{1,2)}$, Manel Ben-Romdhane ${ }^{1)}$, Chiheb Rebai ${ }^{1)}$, Dominique Dallet ${ }^{2)}$ \\ 1) GRESCOM Research Lab., SUP'COM, University of Carthage, Tunisia \\ Cité Technologique des Communications, 2083 El Ghazala, Ariana. \\ manel.benromdhane@supcom.tn \\ 2) IMS Research Lab., University of Bordeaux, Bordeaux INP ENSEIRB-MATMECA, France \\ 351 Cours de la Libération, Bâtiment A31, 33405 Talence Cedex. \\ dominique.dallet@ims-bordeaux.fr
}

\begin{abstract}
Today's bottleneck of signal processing in multistandard software defined radio (SDR) receiver is the analog-to-digital converter (ADC). Therefore, the authors present in this paper the design and simulation results of a programmable parallel frequency band decomposition (FBD) architecture for ADC. The designed parallel architecture is composed of six parallel branches based on discrete-time (DT) $4^{\text {th }}$ order sigma delta modulators using single-bit quantizers. Each branch processes a sub-bandwidth of the received signal. Only needed branches are selected according to the chosen standard. The parallel sigma delta modulators' outputs are handled by a demodulation-based digital reconstruction stage in order to provide the FBD sigma delta-based ADC output signal. The digital reconstruction stage differs from one communication standard to another. In this paper, its design is discussed for the UMTS use case. The objective is to propose a digital reconstruction design with optimized complexity. In fact, the authors propose a comparative study between some configurations of demodulation, decimation and filtering processes. Technical choices and simulation results are discussed. For UMTS use case, the proposed FBD sigma delta-based ADC architecture ensures a computed signal-to-noise ratio (SNR) over $74 \mathrm{~dB}$. Copyright (C) Research Institute for Intelligent Computer Systems, 2016. All rights reserved.
\end{abstract}

Keywords: Sigma delta modulators, frequency band decomposition architecture, software defined radio receiver, wireless communication standards, digital reconstruction.

\section{INTRODUCTION}

Software defined radio (SDR) technology is a state-of-the-art technology solution proposed by scientists to achieve a feasible multistandard receiver [1]. It permits at many coexistent wireless systems to be implemented using common radio platform architecture [1-2]. SDR concept consists in implementing as much as possible of the radio features in software in order to minimize analog circuitry. Therefore, software brings flexibility and adaptability to multistandard receiver while increasing design constraints on the analog-to-digital converter (ADC). In fact, the ADC has to digitize signals from narrowband to wideband with different required dynamic ranges [3-5]. Nonetheless, in the literature, there is no such a fully-integrated ADC that fulfills SDR constraints [6-7]. To overcome this problem, the authors propose the use of parallel architectures based on $\Sigma \Delta$ modulators that ensure high dynamic range while extending signal conversion bandwidth [8].

In fact, $\Sigma \Delta$ modulators present notable interest thanks to their high accuracy. They can reach high resolutions over 16 bits [6]. However, their main drawback is the limited conversion bandwidth due to the noise shaping that requires oversampling [9]. Parallel architectures are therefore used to avoid this limitation. Parallel architectures are time-interleaved sigma delta $(\mathrm{TI} \Sigma \Delta)$ [10], parallel Hadamard sigma delta $(\Pi \Sigma \Delta)[11]$ and frequency band decomposition (FBD) [12-13]. Parallelism using FBD permits extending $\Sigma \Delta$ modulators to wideband applications without the drawbacks of $\operatorname{TI} \Sigma \Delta$ and $\Pi \Sigma \Delta$ architectures [8]. In fact, FBD architecture is not sensitive to gain and offset mismatches [14-15]. FBD is a natural way to extend bandwidth since each $\Sigma \Delta$ modulator of the parallel architecture converts a part of the input signal bandwidth [12-13, 
16]. The parallel modulators' outputs are combined using a digital reconstruction stage to form the final output [16]. Accordingly, the authors take advantage of FBD architecture to design a parallel $\Sigma \Delta$-based ADC architecture intended for an SDR receiver that processes E-GSM, UMTS and IEEE802.11a communication signals. The proposed FBD architecture is composed of six programmable branches based on discrete-time (DT) $4^{\text {th }}$ order $\Sigma \Delta$ modulators [17]. The modulators use single-bit quantization to overcome non-linearity errors introduced by multi-bit quantizers [13].

In the literature, authors propose in $[12-13,16]$ an FBD $\Sigma \Delta$-based ADC architecture that is a generic parallel architecture composed of ten 3rd order continuous-time (CT)- $\Sigma \Delta$ modulators. This architecture operates at $800 \mathrm{MHz}$-sampling frequency with a total conversion bandwidth of 80 $\mathrm{MHz}$ that is uniformly shared between the parallel branches. Each branch processes $8 \mathrm{MHz}$ of signal bandwidth and the architecture realizes a resolution of 13.3 bits. However, in this paper, the novelty is the design of the reconstruction stage of an FBD $\Sigma \Delta$ based ADC architecture for E-GSM, UMTS and IEEE802.11a communication standards. Indeed, the parallel branches are programmable with different sub-bandwidths, where only some branches are active according to the selected standard. The sampling frequency also changes with the chosen standard. The selected branches for a given standard can be reused for the signal digitization of another standard according to the specified sampling frequency and branches' bandwidths configuration. Besides, $\Sigma \Delta$ modulators of the proposed FBD architecture are implemented in discrete-time (DT) instead of continuous-time (CT) as in [12] in order to avoid analog errors.

This paper includes three sections. In section 2, the authors present the system level specifications for the E-GSM, UMTS and IEEE802.11a SDR receiver. Besides, the design results of a programmable FBD $\Sigma \Delta$-based ADC architecture for the multistandard SDR receiver are given. In section 3 , the digital reconstruction stage design of the FBD $\Sigma \Delta$-based ADC is discussed. The authors start from conventional demodulation-based digital reconstruction architecture. Then, modifications are proposed regarding complexity studies. Configurations of two-stage decimation are compared in order to choose the best configuration that provides the lowest complexity. Afterwards, the obtained FBD $\Sigma \Delta$-based ADC with the optimized digital reconstruction stage is implemented on MATLAB/SIMULINK environment and section 4 deals with simulation results. Finally, section 5 draws some conclusions and future works.

\section{FBD $\Sigma \Delta$-BASED ADC ARCHITECTURE FOR SDR RECEIVER}

As today's bottleneck of signal processing in multistandard SDR receiver is the ADC, the authors need to study a novel ADC architecture. Therefore, in this section, SDR receiver system level specifications are presented in the first sub-section. Then, the second sub-section presents FBD $\Sigma \Delta$ based ADC architecture design results.

\subsection{SYSTEM LEVEL SPECIFICATIONS}

In the first hand, the proposed SDR receiver processes E-GSM, UMTS and IEEE802.11a signals [17]. According to these communication standards, design specifications for the multistandard receiver front-end are depicted. In fact, Table 1 summarizes the channel bandwidth and spacing $\left(C h_{B W}\right.$ and $C h_{s p}$, respectively), the reference sensitivity $\left(S_{r e f}\right)$, the signal-to-noise ratio at the receiver input and at the receiver output $\left(S N R_{\text {in }}\right.$ and $S N R_{\text {out }}$, respectively), the analog gain relative to a $13 \mathrm{dBm}$ ADC full scale input $\left(G_{a n a}\right)$, the receiver dynamic range $\left(D R_{i n}\right)$ and the ADC dynamic range $\left(D R_{A D C}\right)$ from which the $\mathrm{ADC}$ resolution $\left(\operatorname{Res}_{A D C}\right)$ is deduced.

Table 1. design specifications for E-GSM/UMTS/IEEE802.11a receiver.

\begin{tabular}{|l|c|c|c|}
\cline { 2 - 4 } \multicolumn{1}{c|}{} & E-GSM & UMTS & $\begin{array}{c}\text { IEEE802.11a } \\
\text { (54 Mbits/s) }\end{array}$ \\
\hline$C h_{B W}(\mathrm{MHz})$ & 0.2 & 3.84 & 16.6 \\
\hline$C h_{s p}(\mathrm{MHz})$ & 0.2 & 5 & 20 \\
\hline$S_{\text {ref }}(\mathrm{dBm})$ & -102 & -117 & -65 \\
\hline$S N R_{\text {in }}(\mathrm{dB})$ & 18.8 & -9 & 36.6 \\
\hline$S N R_{\text {out }}(\mathrm{dB})$ & 9 & -18.2 & 26.6 \\
\hline$G_{\text {ana }}(\mathrm{dB})$ & 28 & 38 & 43 \\
\hline$D R_{\text {in }}(\mathrm{dB})$ & 87 & 92 & 35 \\
\hline$D R_{A D C}(\mathrm{~dB})$ & 96 & 73.8 & 61.8 \\
\hline $\operatorname{Res}_{A D C}(\mathrm{bits})$ & 16 & 12 & 10 \\
\hline
\end{tabular}

In the second hand, a hybrid homodyne/low-IF architecture is presented in [18] for the SDR receiver front-end. The received signals, modulated around the $f_{R F}$ frequency, are first filtered by an RF filter. Second, they are amplified using a low-noise amplifier (LNA). Third, UMTS and IEEE802.11a signals are down-converted by the mixer, which is controlled by the local oscillator (LO), to baseband. However, E-GSM signals are down-converted to a low intermediate frequency which is equal to $100 \mathrm{kHz}$ in order to avoid the flicker noise disturbance [18]. Consequently, the E-GSM channel bandwidth is considered equal to $200 \mathrm{kHz}$ as mentioned in Table 1.

Otherwise, the mixed baseband stage architecture using an FBD-based ADC is presented in Fig. 1. The 
mixer is followed by a non-programmable $6^{\text {th }}$ order Butterworth anti-aliasing filter (AAF) that passes all signals in the IEEE802.11a bandwidth and attenuates blockers around sampling frequency. In this case, there is no need to use an automatic gain control (AGC) block before the ADC [18].Then, the $\mathrm{ADC}$ is designed as a parallel architecture based on FBD architecture and using M DT- $\Sigma \Delta$ modulators. It needs to be followed by a digital reconstruction stage that reconstruct the final output signal from the parallel $\Sigma \Delta$ modulators' output signals while operating decimation and channel selection. The design results of the FBD-based ADC architecture are summarized in the next sub-section.

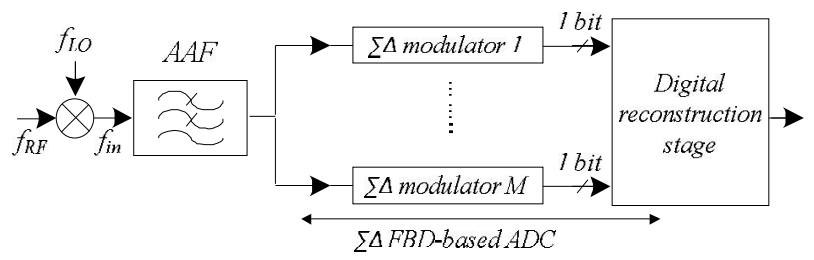

Fig. $1-\Sigma \Delta$ FBD-based mixed baseband architecture.

\subsection{FBD $\Sigma \Delta$-BASED ADC ARCHITECTURE DESIGN RESULTS}

Starting from system level specifications according to the communication standards handled by the SDR receiver, an FBD $\Sigma \Delta$-based ADC architecture design is presented. Design specifications for the multistandard receiver impose high constraints on the ADC stage. In fact, a high dynamic range equal to $96 \mathrm{~dB}$ is required for narrowband E-GSM signals with $200 \mathrm{kHz}$ channel bandwidth in contrast to a weaker required dynamic range of $61.8 \mathrm{~dB}$ for wideband IEEE802.11a signals whose channel bandwidth is equal to $16.6 \mathrm{MHz}$ as shown in Table 1. Therefore, the design of the parallel ADC realizes a trade-off between increasing the sampling frequency while choosing discrete-time $\Sigma \Delta$ modulators but also increasing the number of parallel branches regarding a low-complexity aim, and increasing $\Sigma \Delta$ modulators orders while ensuring their stability [19]. Thus, an FBD design architecture composed of six programmable parallel branches is proposed as presented in Fig. 2 [17]. The first branch uses a $4^{\text {th }}$ order discrete-time (DT) low-pass (LP) $\Sigma \Delta$ modulator. The remaining five other branches are based on $4^{\text {th }}$ order DT band-pass (BP) $\Sigma \Delta$ modulators. The $\Sigma \Delta$ modulator order is defined as the number of integrators or resonators, $p$, for LP or BP $\Sigma \Delta$ modulators, respectively. Since the designed FBD architecture is composed of both LP and BP $\Sigma \Delta$ modulators, the authors designate $p$ as $\Sigma \Delta$ modulator order [17].

The proposed design is programmable since according to the selected communication standard, only some branches are active. Sampling frequency choice also modifies operating $\Sigma \Delta$ modulators' subbandwidths of the selected branches. This is defined as in the branch frequency division plan presented by Fig. 3 [17].

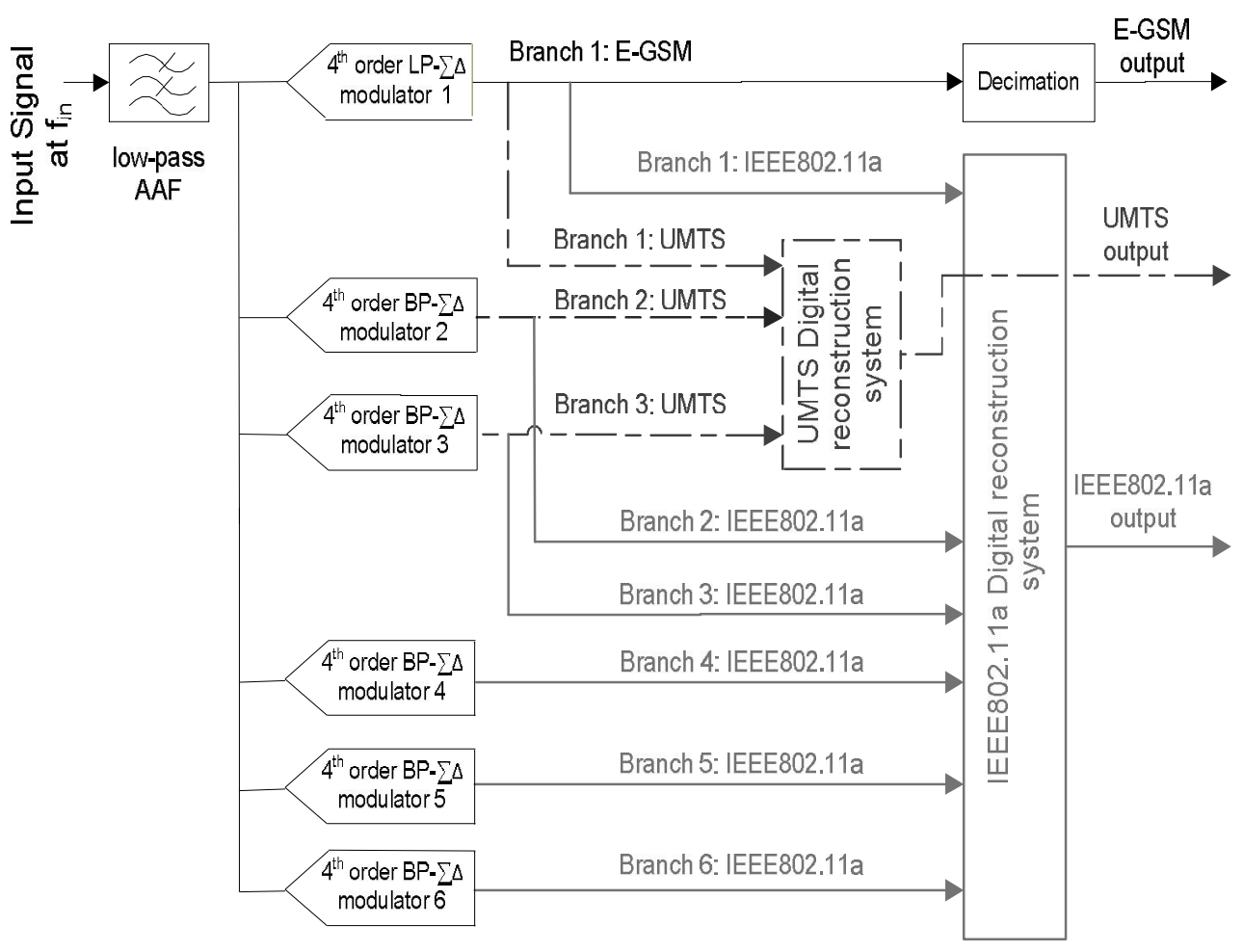

Fig. 2 - Designed FBD $\Sigma \Delta$-based ADC architecture. 
Indeed, the branches that digitize a given standard's signals can be reused to digitize another standard's signals. This leads to a final flexible architecture. For this architecture, sampling frequencies are lower than $100 \mathrm{MHz}$ to permit synthesizing the $\Sigma \Delta$ modulators of the designed FBD architecture in discrete-time domain and thus avoiding analog errors. In fact, the discrete-time technology limits sampling frequencies for the $\Sigma \Delta$ modulators [6].

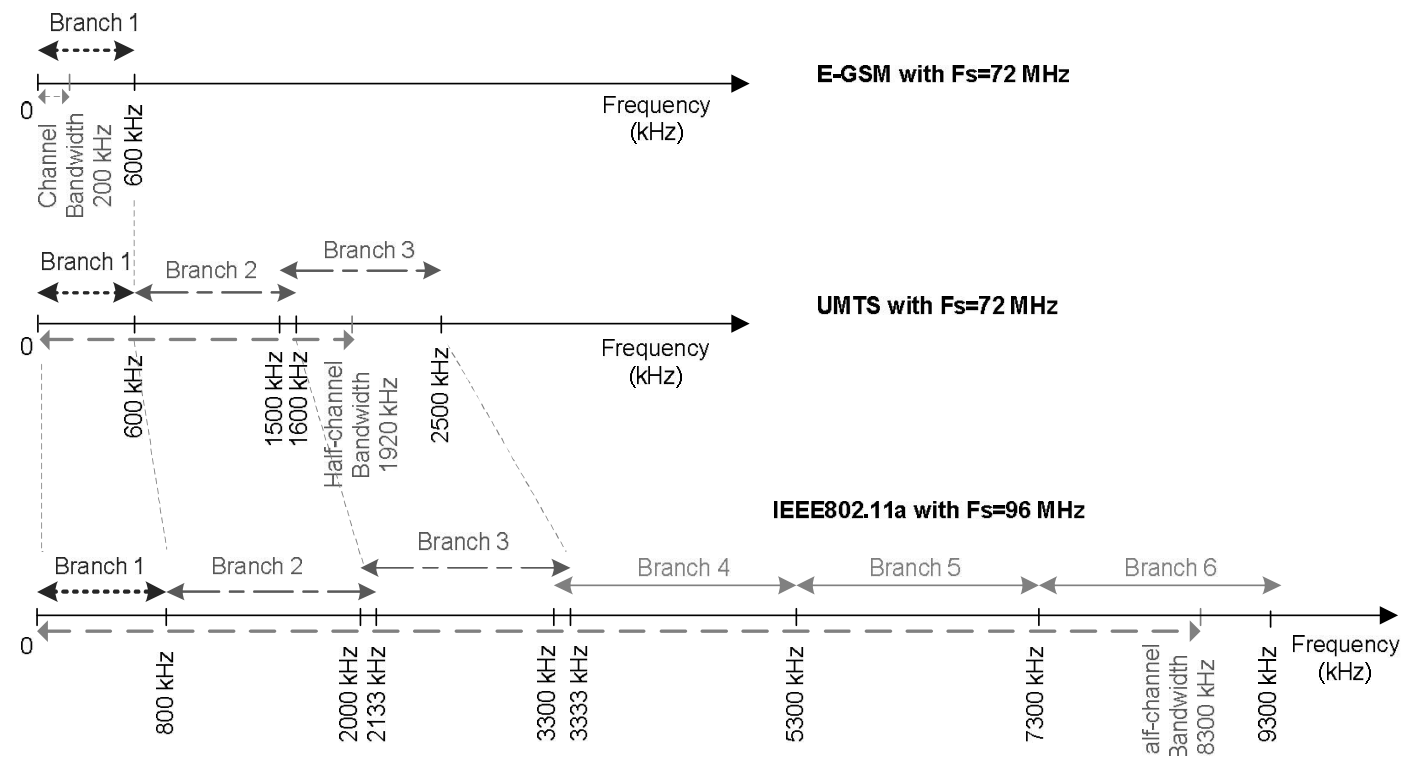

Fig. 3 - Branch frequency division plan.

A digital reconstruction stage is required to combine the parallel $\Sigma \Delta$ modulators outputs of the FBD architecture in order to recover the final output signal. The design of this stage is discussed in the next section.

\section{DIGITAL RECONSTRUCTION STAGE DESIGN}

In the literature, for digital reconstruction process, there are two main solutions which are the direct reconstruction and the demodulation-based digital reconstruction [12]. The first solution is based on BP filters placed at the parallel $\Sigma \Delta$ modulators' outputs. These filters permit selecting useful signals in selected sub-bandwidths. Then, selected parallel signals are summed using an adder that is followed by a decimation operation. The reconstructed final output is therefore obtained. The main drawback of this direct reconstruction is that the BP filters present high complexity since they operate at the oversampling frequency of the $\Sigma \Delta$ modulators [12]. The second approach for digital reconstruction based on demodulation offer a solution to this problem.

In fact, the BP $\Sigma \Delta$ modulators' output signals are firstly down-converted to baseband thanks to a complex demodulation operation. For the first branch, since the $\Sigma \Delta$ modulator is LP, there is no need to perform demodulation. Secondly, after demodulation of BP modulators' output signals, a decimation process is applied to the baseband signals before their selection by LP filters. The LP filters in the demodulation-based reconstruction approach present lower complexity compared with BP filters of the direct reconstruction approach since they operate at Nyquist sampling frequency. Consequently, in this section, the authors take advantage of the demodulation-based digital reconstruction approach to propose a design for the digital reconstruction stage intended for the proposed FBD $\Sigma \Delta$-based ADC architecture. The UMTS standard is chosen as a use case study. First sub-section discusses the demodulation position after the $\Sigma \Delta$ modulators. However, in the second sub-section, the authors compare the configurations of two-stage decimation in order to decide on the best configuration that provides the lowest complexity.

\subsection{DEMODULATION POSITION STUDY}

In conventional demodulation-based digital reconstruction architecture, the BP $\Sigma \Delta$ modulators' output signals are first frequency down-converted to baseband using a complex digital demodulation. This operation consists in multiplying modulators' output signals by a complex sequence $m_{k}[n]$ as given by (1) where $f_{c_{k} k}$ is the $k^{\text {th }}$ central frequency of the $k^{\text {th }}$ branch sub-bandwidth, $T_{s}$ is the oversampling period and $n$ is a positive integer [20].

$$
m_{k}[n]=e^{-2 i \pi f_{c_{-} k} n T_{s}}
$$


Second, after demodulation, a decimation process is mandatory for the baseband signals that are at the oversampling frequency, $F_{s}$, which is equal to $1 / T_{s}$. The oversampling frequency, $F_{s}$, corresponds to the sampling frequency of the DT $\Sigma \Delta$ modulators. The decimation process permits the sampling frequency reduction of oversampled baseband signals to bring them to the Nyquist frequency which is defined as the double of the channel bandwidth, ChBW. The global decimation factor is equal to the global oversampling ratio, $O S R$, defined as the oversampling frequency, Fs, out of the Nyquist frequency. For the UMTS use case, it is chosen equal to 16 . Thus, the UMTS signals are digitized at a Nyquist frequency equal to $4.5 \mathrm{MHz}$. This Nyquist frequency is comprised between the UMTS channel bandwidth, $C h_{B W}$, and channel spacing $C h_{s p}$, that are presented in Table 1.

Besides, to implement complex digital demodulation on MATLAB/SIMULINK environment or also for hardware implementation in future works, it is required to use in-phase and quadrature (I/Q) demodulation as illustrated by Fig. 4. Demodulation filters are then needed for I and Q signals to filter out the undesired component of the digital mixer output. After that, to reduce the complexity of decimation filters, a two-stage

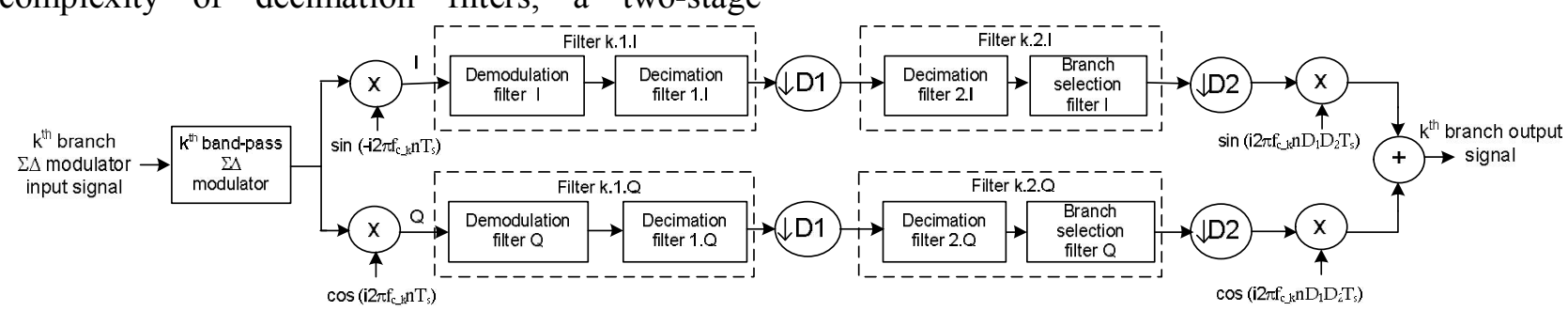

Fig. $4-k^{\text {th }}$ branch architecture, $2 \leq k \leq M$, of the demodulation-based digital reconstruction stage where demodulation is after the $\Sigma \Delta$ modulator.

Table 2. UMTS Second branch demodulation filter design: parameters and complexity computation.

\begin{tabular}{|l|c|}
\cline { 2 - 2 } \multicolumn{1}{c|}{} & $\begin{array}{c}\text { Demodulation } \\
\text { filter }\end{array}$ \\
\hline Cutoff frequency (MHz) & 0.5 \\
\hline In-band maximal attenuation (dB) & 0.1 \\
\hline Sampling frequency (MHz) & 72 \\
\hline Rejection frequency (MHz) & 1.7 \\
\hline $\begin{array}{l}\text { Maximum between quantization } \\
\text { noise level and blocker level (dBm) }\end{array}$ & -28 \\
\hline Minimal attenuation (dB) & 32.8 \\
\hline Filter order & 114 \\
\hline$M P S\left(\mathrm{x} 10^{6}\right)$ & 8208 \\
\hline
\end{tabular}

These results are identical for both of I and Q demodulation filters. It is shown in Table 2 that the decimation process is operated. Each decimation stage is composed of decimation filter and downsampling at rates $D_{1}$ or $D_{2}$. In the first decimation stage, the authors propose to combine demodulation and decimation filters in order to obtain Filter k.1.I and Filter k.1.Q for the $k^{\text {th }}$ branch as shown in Fig. 4. In the second decimation stage, the authors propose to combine branch selection and decimation filters in order to obtain Filter k.2.I and Filter k.2.Q for the $k^{\text {th }}$ branch as shown in Fig. 4. Indeed, branch selection filter is required to select the branch sub-bandwidth to attenuate out-of-band $\Sigma \Delta$ modulator quantization noise. After second stage demodulation, since the signals at the parallel branches are in baseband, it is mandatory to perform frequency up-conversion using modulation. The signals are therefore upconverted from baseband to around the central frequency of the adequate branch sub-bandwidth at the Nyquist frequency. Then, the signals are added to obtain the $k^{\text {th }}$ branch output signal.

To discuss the demodulation position, the authors start with the demodulation filter design. Table 2 summarizes, for example, demodulation filter design parameters as well as its complexity computation in terms of multiplications per second, MPS, for the second branch of UMTS use case. 


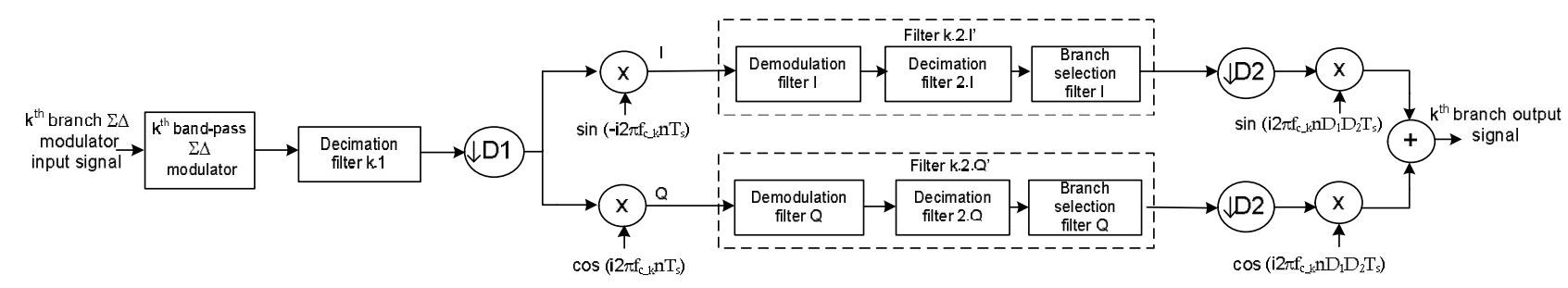

Fig. $5-k^{\text {th }}$ branch architecture, $2 \leq k \leq M$, of the demodulation-based digital reconstruction stage where demodulation is after the first decimation stage.

\subsection{COMPARISON OF TWO-STAGE DECIMATION CONFIGURATIONS}

For the 16-global decimation factor, the twostage decimation configurations $D_{I} \times D_{2}$ are $8 \times 2,4 \times 4$ and $2 \times 8$. They are studied in order to compare their complexities and choose the less complex one. The results of Filter 2.1, Filter 2.2.I' and Filter 2.2.Q' design parameters, complexity computation and signal-to-noise ratio $(S N R)$ for $8 \times 2,4 \times 4$ and $2 \times 8$ configurations are presented in Table 3, Table 4 and Table 5, respectively. From theses tables, it is shown that the less complex two-stage decimation configuration is the $8 \times 2$ where the first stage ensures decimation by a factor of 8 and the second by 2 . In fact, the total MPS is equal to $2763 \times 10^{6}$. However, the $4 \times 4$ and $2 \times 8$ configurations require much more total MPS that are equal to $3780 \times 10^{6}$ and $11016 \times 10^{6}$, respectively.

Table 3. Filter 2.1, Filter 2.2.I' and Filter 2.2.Q' design for UMTS Second branch: parameters, complexity computation and SNR for 8x2 decimation configuration.

\begin{tabular}{|l|c|c|}
\cline { 2 - 3 } \multicolumn{1}{c|}{} & Filter 2.1 & $\begin{array}{c}\text { Filter 2.2.I' } \\
\text { Filter 2.2.Q' }\end{array}$ \\
\hline Cutoff frequency (MHz) & 1.6 & 0.3 \\
\hline In-band maximal attenuation (dB) & 0.1 & 0.1 \\
\hline Sampling frequency (MHz) & 72 & 9 \\
\hline Rejection frequency (MHz) & 7.4 & 0.7 \\
\hline Maximum between quantization noise & $-18.1 \mathrm{dBm} @ 7.4 \mathrm{MHz}$ & $-19 \mathrm{dBm} @ 0.7 \mathrm{MHz}$ \\
level and blocker level (dBm) & $-12.7 \mathrm{dBm} @ 16.4 \mathrm{MHz}$ & $79.8 \mathrm{~dB} @ 0.7 \mathrm{MHz}$ \\
\hline Minimal attenuation $(\mathrm{dB})$ & $42.7 \mathrm{~dB} @ 7.4 \mathrm{MHz}$ & 75 \\
\hline Filter order & $48.1 \mathrm{~dB} @ 16.4 \mathrm{MHz}$ & 675 \\
\hline$M P S\left(\mathrm{x} 10^{6}\right)$ & 29 & \multicolumn{2}{|c|}{2763} \\
\hline Total $M P S\left(\mathrm{x} 10^{6}\right)$ & 2088 & \multicolumn{2}{|c|}{81.08} \\
\hline$S N R$ at the second branch output $(\mathrm{dB})$ & \multicolumn{2}{|c|}{} \\
\hline
\end{tabular}

Table 4. Filter 2.1, Filter 2.2.I' and Filter 2.2.Q' design for UMTS Second branch: parameters, complexity computation and SNR for $4 \times 4$ decimation configuration.

\begin{tabular}{|l|c|c|}
\cline { 2 - 3 } \multicolumn{1}{c|}{} & Filter 2.1 & $\begin{array}{c}\text { Filter 2.2.I' } \\
\text { Filter 2.2.Q' }\end{array}$ \\
\hline Cutoff frequency (MHz) & 1.6 & 0.3 \\
\hline In-band maximal attenuation (dB) & 0.1 & 0.1 \\
\hline Sampling frequency $(\mathrm{MHz})$ & 72 & 18 \\
\hline Rejection frequency $(\mathrm{MHz})$ & 16.4 & 0.7 \\
\hline $\begin{array}{l}\text { Maximum between quantization noise level } \\
\text { and blocker level (dBm) }\end{array}$ & -12.7 & -19 \\
\hline Minimal attenuation $(\mathrm{dB})$ & 86.1 & 79.8 \\
\hline Filter order & 15 & 150 \\
\hline MPS $\left(\mathrm{x} 10^{6}\right)$ & 1080 & 2700 \\
\hline Total MPS $(\mathrm{x} \mathrm{10})$ & \multicolumn{2}{|c|}{3780} \\
\hline SNR at the second branch output $(\mathrm{dB})$ & 76.46 \\
\hline
\end{tabular}


Table 5. Filter 2.1, Filter 2.2.I' and Filter 2.2.Q' design for UMTS Second branch: parameters, complexity computation and SNR for $2 \times 8$ decimation configuration.

\begin{tabular}{|l|c|c|}
\cline { 2 - 3 } \multicolumn{1}{c|}{} & Filter 2.1 & $\begin{array}{c}\text { Filter 2.2.I' } \\
\text { Filter 2.2.Q' }\end{array}$ \\
\hline Cutoff frequency $(\mathrm{MHz})$ & 1.6 & 0.3 \\
\hline In-band maximal attenuation $(\mathrm{dB})$ & 0.1 & 0.1 \\
\hline Sampling frequency $(\mathrm{MHz})$ & 72 & 36 \\
\hline Rejection frequency $(\mathrm{MHz})$ & 34.4 & 0.7 \\
\hline $\begin{array}{l}\text { Maximum between quantization noise } \\
\text { level and blocker level }(\mathrm{dBm})\end{array}$ & -19 & -19 \\
\hline Minimal attenuation $(\mathrm{dB})$ & 79.8 & 79.8 \\
\hline Filter order & 3 & 300 \\
\hline MPS $\left(\mathrm{x} 10^{6}\right)$ & 216 & 10800 \\
\hline Total MPS $\left(\mathrm{x} 10^{6}\right)$ & \multicolumn{2}{|c|}{11016} \\
\hline SNR at the second branch output $(\mathrm{dB})$ & \multicolumn{2}{|c|}{74.28} \\
\hline
\end{tabular}

Moreover, the authors implement the second branch with $8 \times 2,4 \times 4$ and $2 \times 8$ decimation configurations on MATLAB/SIMULINK environment. The choice of $8 \times 2$ decimation configuration is confirmed thanks to SNR measurements of the second branch output signal. Indeed, SNR are equal to $81.08 \mathrm{~dB}, 76.46 \mathrm{~dB}$ and $74.28 \mathrm{~dB}$ for $8 \times 2,4 \times 4$ and $2 \times 8$ decimation configurations, respectively. The signals from the parallel branches are finally summed to form the overall final output signal of the FBD $\Sigma \Delta$-based $\mathrm{ADC}$ for the UMTS use case. This architecture is illustrated in Fig. 6.

The next section deals with simulation results of the designed FBD $\Sigma \Delta$-based ADC architecture using MATLAB/SIMULINK environment.

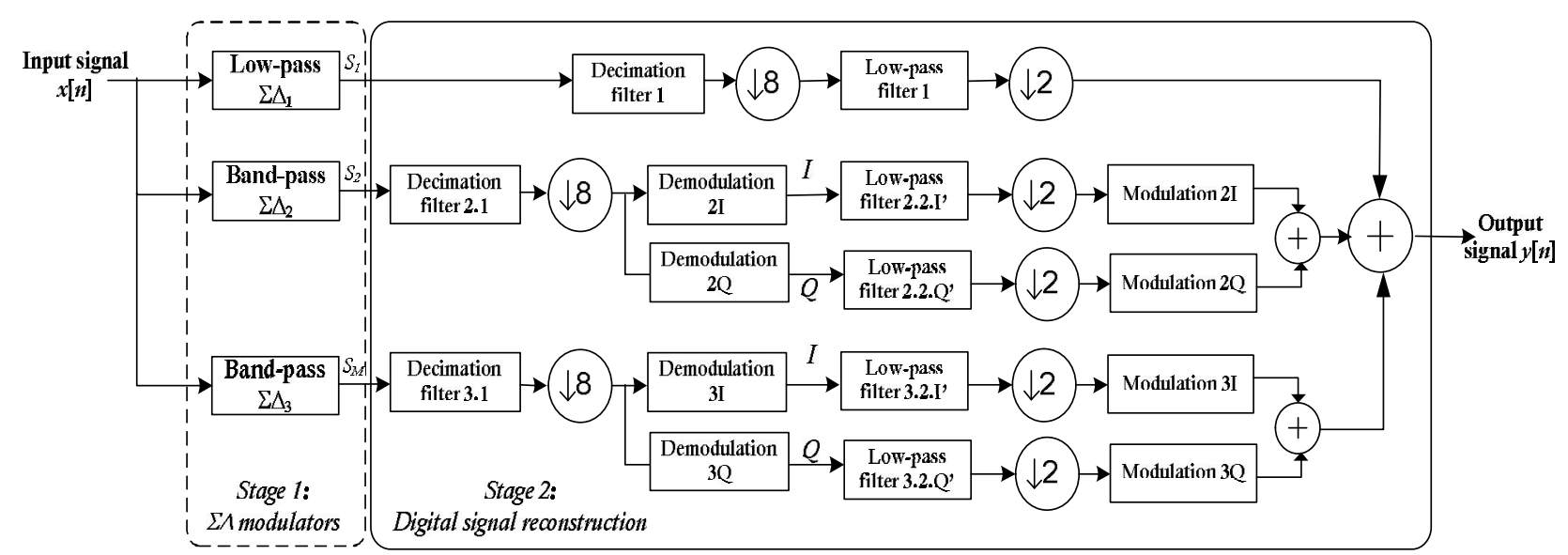

Fig. 6. Proposed demodulation-based digital reconstruction for the FBD $\Sigma \Delta$-based ADC architecture for the UMTS use case.

\section{SIMULATION RESULTS}

In this section, the authors start simulation results with SNR measurements for a single-tone input signal at a frequency varying in the UMTS channel bandwidth $\left(C h_{B W} / 2\right)$. The computed SNR of the FBD $\Sigma \Delta$-based ADC output signal are presented in Fig. 7. The computed SNR values vary from $68 \mathrm{~dB}$ to 73.7 $\mathrm{dB}$. Therefore, it is important to test the designed FBD $\Sigma \Delta$-based ADC architecture by at least a signal in the operating sub-bandwidth of each branch in parallel to ensure required dynamic range. In practical case, the received modulated signal occupies all the channel bandwidth.

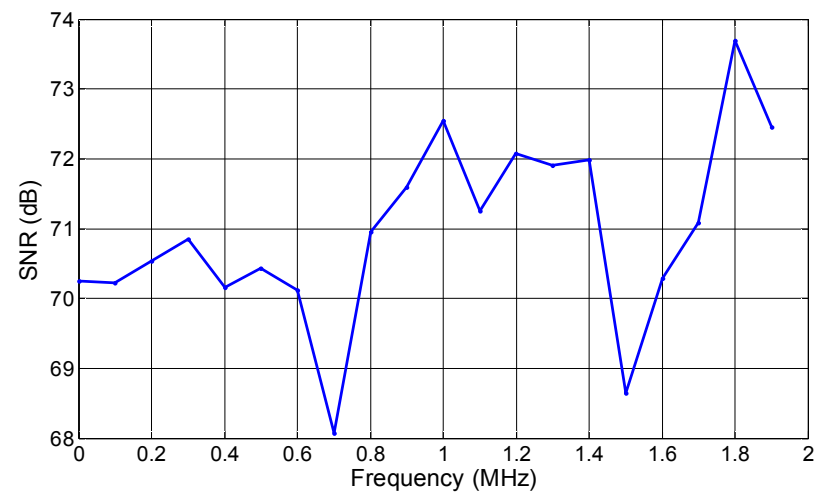

Fig. 7. Computed SNR in UMTS channel bandwidth versus input frequency for a single-tone input signal. 
Moreover, simulation results are realized by applying a multi-tone signal which is composed of three sine-wave signals. This signal is applied at the input of the FBD architecture. The sine-wave frequencies values are $300 \mathrm{kHz}, 800 \mathrm{kHz}$ and 1900 $\mathrm{kHz}$ which belong to the first, second and third subbandwidth of the UMTS standard, respectively. The sine-wave normalized amplitudes are set at 0.45 . The normalized amplitude is defined as the input amplitude out of the power supply voltage. The recombined final output signal spectrum of the FBD $\Sigma \Delta$-based ADC which is realized in MATLAB/SIMULINK model is presented in Fig. 8. The SNR of this output signal is computed. The obtained value from the simulation results is equal to $74.63 \mathrm{~dB}$ which satisfies the $73.8-\mathrm{dB}$ required dynamic range for UMTS standard. The effective resolution can be deduced from the SNR and reaches 12.1 bits.

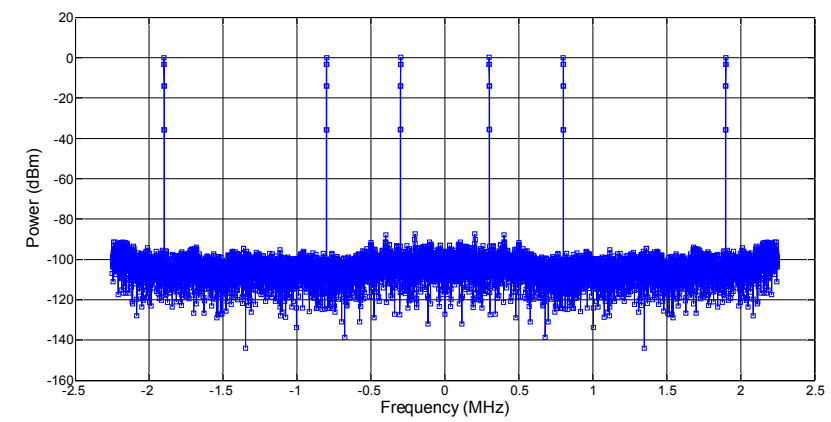

Fig. 8. Spectrum of the FBD $\Sigma \Delta$-based ADC output signal for UMTS use case.

\section{CONCLUSION}

In this paper, the demodulation-based digital reconstruction stage design for the frequency band decomposition $\Sigma \Delta$-based analog-to-digital converter is proposed. This parallel $\mathrm{ADC}$ architecture design is intended for multistandard software radio receiver which processes E-GSM, UMTS and IEEE802.11a communication signals. The designed FBD model for UMTS use case, is implemented on MATLAB/SIMULINK environment. The computed signal-to noise ratio of the output signal spectrum is equal to $74.63 \mathrm{~dB}$ which is higher than the required UMTS dynamic range. The proposed parallel ADC is programmable and flexible. In future work, the MATLAB/SIMULINK simulated model will be hardware implemented and tested on FPGA.

\section{REFERENCES}

[1] J. Mitola, Software radios: survey, critical evaluation and future directions, IEEE Aerospace and Electronic Systems Magazine, (8) 4 (1993), pp. 25-36.
[2] W. H. W. Tuttlebee, Software-defined radio: facets of a developing technology, IEEE Personal Comm., (6) 2 (1999), pp. 38-44.

[3] GSM Technical Specification, Radio Transmission and Reception (GSM 05.05), European Telecommunications Standards Institute, 1996.

[4] UMTS, User Equipment (UE) Radio Transmission and Reception (FDD), 3GPP TS 25.101, Version 6.19.0 Release 6, European Telecommunications Standards Institute, 2009.

[5] IEEE802.11a, Telecommunications and information exchange between systems Local and metropolitan area networks - Specific requirements Part 11: Wireless LAN Medium Access Control (MAC) and Physical Layer (PHY) Specifications, in IEEE Std 802.11-2012 (Revision of IEEE Std 802.11-2007), pp.12793, mars 2012.

[6] J. M. de la Rosa, An empirical and statistical comparison of state-of-the-art sigma-delta modulators, in proceedings of the IEEE International Symposium on Circ. and Syst., (May 2013), pp. 825-828.

[7] J. M. De la Rosa, R. del Rio, CMOS SigmaDelta Converters Practical Design Guide, IEEE Press, A John Wiley \& Sons, Ltd., Publication, 2013.

[8] A. Eshraghi, T. Fiez, A comparative analysis of parallel delta-sigma ADC architectures, IEEE Trans. Circuits and Syst. I: Regular Papers, (51) 3 (2004), pp. 450-458.

[9] Y. Xiaolong, Wideband Sigma-Delta Modulators, Phd Thesis, KTH Information and Communication Technology, Stockholm, Sweden, 2010.

[10] W. Black, D. Hodges, Time interleaved converter arrays, IEEE J. of Solid-State Circ., (15) 6 (1980), pp. 1022-1029.

[11] I. Galton, H.T. Jensen, Delta-sigma modulator based A/D conversion without oversampling, IEEE Trans. on Circ. and Syst. II: Analog and Digital Signal Process., (42) 12 (1995), pp. 773-784.

[12] P. Benabes, A. Beydoun, M. Javidan, Frequency-band-decomposition converters using continuous-time $\Sigma \Delta \mathrm{A} / \mathrm{D}$ modulators, in Proceedings of the IEEE North-East Workshop on Circ. and Syst. and TAISA Conf., (June 2009).

[13] P. Benabes, Extended Frequency-BandDecomposition Sigma-Delta A/D converter, Analog Integr. Circ. Process., 2009.

[14] A. Eshraghi, T. Fiez, A comparison of three parallel $\Sigma \Delta$ A/D converters, in Proceedings of the IEEE International Symposium on Circ. and Syst., (May 1996), pp. 517-520. 
[15] A. Blad, H. Johansson, P. Lowenborg, A general formulation of analog-to-digital converters using parallel sigma-delta modulators and modulation sequences, in Proceedings of the IEEE Asia Pacific Conference on Circuits and Systems, (December 2006), pp. 438-441.

[16] A. Beydoun, P. Benabes, Bandpass/wideband ADC architecture using parallel delta sigma modulators, in Proceedings of the European Signal Processing Conference, (September 2006).

[17] R. Lahouli, M. Ben-Romdhane, C. Rebai, D. Dallet, Towards flexible parallel sigma delta modulator for software defined receiver, in Proceedings of the IEEE International Instrumentations and Measurement Technology Conference, (May 2014).

[18] M. Ben-Romdhane et al., Nonuniformly controlled analog-to-digital converter for SDR multistandard radio receiver, IEEE Trans. Circuits and Syst. II Brief Papers, (58) 12 (2011), pp. 862-866.

[19] R. Schreier, An empirical study of high-order single-bit delta-sigma modulators, IEEE Trans. Circ. and Syst. II: Analog and Digital Signal Process., (40) 8 (1993), pp. 461-466.

[20] R. Lahouli, M. Ben-Romdhane, C. Rebai, D. Dallet, Digital reconstruction stage of the FBD $\Sigma \Delta$-based ADC architecture for multistandard receiver, in Proceedings of the 20th IMEKO TC4 Int. Workshop on ADC Modelling and Testing Research on Electric and Electronic Meas. for the Economic Upturn, (September 2014).

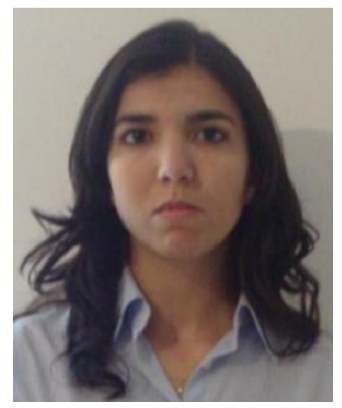

Rihab Lahouli received her Engineer degree in telecommunications from ESPRIT in 2010. In 2005, she received her master degree in telecommunications - speciality: Circuits \& Systems for Telecommunications Networks from the Higher School of Communications (SUP'COM) of the University of Carthage in Tunis-Tunisia. From 2011, she is preparing her PhD degree in Electronics and Telecommunications engineering in co-supervision between University of Bordeaux and SUP'COM.

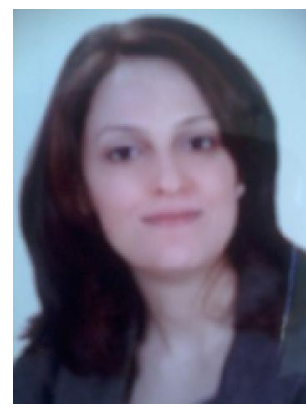

Manel Ben-Romdhane received her Engineer degree in telecommunications from the Higher School of Communications of Tunis (SUP'COM) in 2004. In 2005, she received her master degree in telecommunications - speciality: Circuits \& Systems for Telecommunications Networks from SUP'COM of the University of Carthage in Tunis, Tunisia, and her master degree in digital telecommunication systems from the Ecole Nationale Supérieure des Télécommunications de Paris (TELECOM ParisTech) and the Université de Pierre et Marie Curie in Paris (UPMC). In 2009, she obtained her $\mathrm{PhD}$ degree in Electronics and Telecommunications engineering in co-supervision between TELECOM Paris-Tech and SUP'COM. Her PhD is subscribed in a CMCU (Comité Mixte de Coopération Universitaire) project titled "Reconfigurable $R F$ receiver for Software Defined Radio applications". Since 2005, she has been a member of GRESCOM research Laboratory. In 2007, she joined the Institut Supérieur de Biotechnologie de Sfax, Tunisia, where she was Assistant. In 2009, she joined the Faculté des Sciences de Bizerte, Tunisia, as Assistant Professor. Since september 2014, she has joined SUP'COM. Her research activities are in the area of mixed signal, circuits and systems for radio communications.

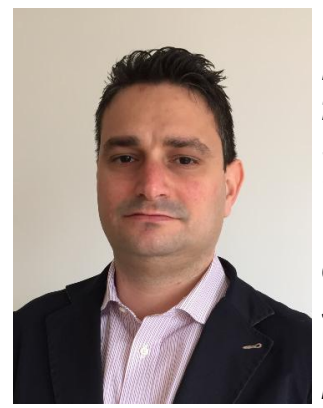

Chiheb REBAl received the Electrical Engineering degree from National Engineering School of Sfax (Tunisia) in 1999 with honors (Rank \#1 in Tunisia), the M.S. and Ph.D. degrees of Microelectronic sciences with honors from University of Bordeaux (France), in 2000 and 2002, respectively and the Habilitation degree in communication and information technologies from Higher School of Communications of Tunis (Tunisia) in 2009. He was at IMS laboratory in Bordeaux (France) from 1999 to 2002 as member of technical staff in the advanced data converters team. He was a visiting researcher at ST-Microelectronics Grenoble (France) in standards IC design group from 2002 to 2003. Since 2004, he has been with Electronics, Physics and Propagation Department of Higher School of Communications of Tunis (SUP'COM) where he is a Full Professor. From 2009, he is a research Director and a founding member of GRES'COM (Green and 
Smart Communication Systems) research Laboratory, managing the MS'CAS (Mixed Signal Circuits and Systems for communication) research group and working on the Development of new topologies for software radio systems and the definition of cognitive processing techniques with a special focus on the design and implementation of innovative solutions for processing and intelligent communication relative to e-health and Smart Grid. Since 2004, he is a senior technology consultant in a various international industrial companies working for clients in R\&D environment across Asia, Europe and America. He has a good understanding with relevant hands-on expertise in Business Process Outsourcing, Information Technology Outsourcing, industry, spin-off design/establishment. He is leading awarded research projects in emergent ICT sectors as Lead project investigator (LPI), acting as TCP member in various scientific top scientific journals and industrial conferences and serving as panelist for many accountable research funding agencies. His research led to over 100 academics publications (journals \& conferences) and 15 industrial and market studies.

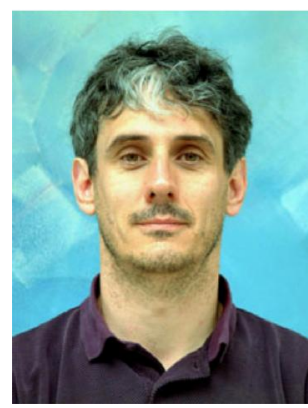

Dominique DALLET is Full Professor at Bordeaux INP and researcher at the IMS Laboratory. His teaching activities mainly concern all the topics around digital electronic design. His research activities focus on the performance improvement of the analog and mixed circuits by means on digital signal processsing algorithms as well as digital implementation. This requires skills in modeling, parameter estimation methods, signal processing algorithms and implementation on digital electronic target (FPGA and ASIC). Currently he is working on analog to digital conversion systems and power amplifiers. $\mathrm{He}$ is involved in IMEKO society as chairman of the technical committee TC4. He is also involved in Chapter IEEE Instrumentation and Measurement through his participation in various standards and he belongs to the new IEEE France chapter on Instrumentation and Measurement France. 